\title{
Influence of outdoor rearing and oleic acid supplementation on lipid characteristics of muscle and adipose tissues from obese Alentejano pigs
}

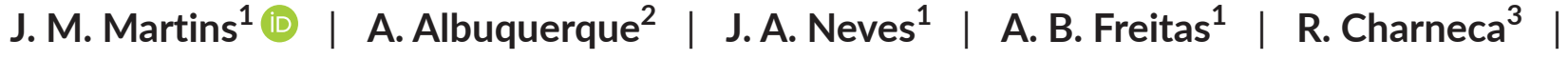 \\ J. L. Tirapicos ${ }^{3}$
}

\author{
${ }^{1}$ Departamento de Zootecnia, ICAAM - \\ Instituto de Ciências Agrárias e Ambientais \\ Mediterrânicas, Universidade de Évora, Évora, \\ Portugal \\ ${ }^{2}$ ICAAM - Instituto de Ciências Agrárias e \\ Ambientais Mediterrânicas, Universidade de \\ Évora, Évora, Portugal \\ ${ }^{3}$ Departamento de Medicina \\ Veterinária, ICAAM - Instituto de \\ Ciências Agrárias e Ambientais \\ Mediterrânicas, Universidade de Évora, Évora, \\ Portugal

\section{Correspondence} \\ J. M. Martins, Departamento de Zootecnia, \\ Universidade de Évora, Évora, Portugal. \\ Email: jmartins@uevora.pt.

\section{Funding information} \\ FCT-Foundation for Science and \\ Technology, Grant/Award Number: UID/ \\ AGR/00115/2013; AGRO research \\ programme
}

\begin{abstract}
Summary
Data evaluating the effect of oleic-acid-enriched diets and outdoor rearing (with access to pasture) on the quality of Alentejano ( $A L$ ) pork and fat are limited. The aim of this work was to study the effects of feeding low (LO) or high oleic acid (HO) diets to AL pigs reared in individual pens (IND) or outdoors (OUT) with access to pasture. Biceps femoris and dorsal subcutaneous fat (DSF) samples were collected to analyse chemical composition and lipids fatty acid (FA) profile. The expression of seven genes involved in lipogenesis, lipolysis and FA transport, as well as two transcription factors were also evaluated in the muscle. B. femoris and DSF presented lower lipid content in OUT-reared than in IND-reared pigs. Two genes involved in lipogenesis, ACACA and FASN, were under-expressed in B. femoris from OUT-reared pigs, while two involved in lipolysis, LPL and HSL were over-expressed. HO diet also significantly reduced FASN expression. Both rearing system and diet affected the FA profile of tissues, particularly DSF. Overall, OUT-reared pigs presented lower n6 to n3 FA ratios in all tissues, and lower monounsaturated (MUFA) and higher polyunsaturated fatty acids (PUFA) content in DSF. When compared to LO-fed ones, HO-fed pigs presented higher oleic acid proportions in all tissues, and lower SFA, higher MUFA and PUFA levels, as well as lower saturation, atherogenic and thrombogenic indexes in DSF. This study shows that rearing system and oleic acid supplementation have complementary effects and influence the nutritional quality of pork and DSF.
\end{abstract}

\section{KEYWORDS}

Alentejano pig, dorsal subcutaneous fat, gene expression, intramuscular fatty acids, oleic acid, rearing system

\section{1 | INTRODUCTION}

The Alentejano (AL) pig breed from the southern region of Portugal, genetically similar to the Iberian pig, has been traditionally reared for centuries under free-range conditions and finished on oak woodland pasture during autumn/winter months. This obese pig (FernándezFígares, Conde-Aguilera, Nieto, Lachica, \& Aguilera, 2008), with slow growth rates and precocious lipogenic activity, is slaughtered at heavy

\footnotetext{
Abbreviations: ACACA, acetyl-CoA carboxylase; AL, alentejano; BW, body weight; DSF, dorsal subcutaneous fat; DSFi, dorsal subcutaneous fat inner; DSFo, dorsal subcutaneous fat outer; FA, fatty acid; FABP3, heart-type fatty acid binding protein; FASN, fatty acid synthase; HO, high oleic acid; HPRT1, hypoxanthine phosphoribosyltransferase 1; HSL, hormone-sensitive lipase; IMF, intramuscular fat; IND, individual pens; LO, low oleic acid; LPL, lipoprotein lipase; MCPT1, muscle-type carnitine palmitoyltransferase 1; MUFA, monounsaturated fatty acids; OUT, outdoor; PPARA, peroxisome proliferator-activated receptor alpha; PPARG, peroxisome proliferator-activated receptor gamma; PUFA, polyunsaturated fatty acids; SCD, Stearoyl-CoA desaturase; SFA, saturated fatty acids.
} 